\title{
ENVELHECIMENTO CEREBRAL
}

\section{O PROBLEMA DOS LIMITES ENTRE O NORMAL E O PATOLÓGICO}

\author{
BENITO PEREIRA DAMASCENO*
}

\begin{abstract}
RESUMO - O autor relata três casos de pseudodemência e discute o problema dos limites entre o normal e o patológico no sujeito idoso. O envelhecimento cerebral acompanha-se de alterações mentais e neuropatológicas superponíveis às da demência de Alzheimer (DA), levando a problemas de diagnóstico diferencial. Ainda não existe um marcador biológico seguro de DA e os testes psicométricos podem dar resultados falso-positivos ou falso-negativos. No idoso normal, o declínio da memória operacional e memória secundária é maior que o das memórias primária e terciária, de modo similar ao encontrado nas fases iniciais da DA. Os testes de evocação retardada de lista de palavras, memória lógica, pensamento categórico, destreza visuo-motora-espacial e o Teste de Nomeação de Boston têm sido apontados como os mais discriminativos entre demência incipiente e normalidade. Na neuroimagem, os achados de atrofia ou hipoperfusão em regiões entorrinais-hipocampais ou temporo-parietais são sugestivos de DA, mas podem estar ausentes nas fases iniciais desta doença. $\mathrm{O}$ autor conclui sugerindo passos decisivos para o diagnóstico diferencial: avaliação neuropsicológica e comportamental abrangente (com levantamento do nível pré-mórbido de funcionamento cognitivo e sócio-ocupacional do paciente), bem como exames laboratoriais e de neuroimagem; e, se preciso, reavaliação após 4 a 6 meses, para verificar a consistência dos achados.
\end{abstract}

PALAVRAS-CHAVE: envelhecimento cerebral, demência, testes neuropsicológicos.

\section{Brain ageing: problems of differential diagnosis between normal and pathologic}

ABSTRACT - The author reports three cases of pseudodementia and discuss the difficulties in establishing limits between normality and illness in the elderly. The mental and neuropathological changes that accompany the normal ageing of the brain are similar to those of early Alzheimer's dementia (AD). These similarities often lead to difficulties in the differential diagnosis, hence the search for consensus criteria. The decline of working and secondary memory is greater than that of primary and tertiary memory, as is found in AD. On the other hand, tests of delayed recall of 10 to 15 unrelated words, logical memory, categorical thinking, visuo-motor-spatial skills, and Boston Naming Test have been pointed out as the most discriminative. Neuroimaging findings of atrophy or hipoperfusion in the entorhinal-hippocampal or temporo-parietal regions are suggestive of DA, but they can be lacking in the early stages of this disease. In the conclusion, the author suggests the diagnostic process should be based on a comprehensive neuropsychological and behavioral evaluation (including a survey of the subject's premorbid level of cognitive and socio-occupational functioning), supplemented by neuroimaging and laboratory tests. If inconclusive, the whole evaluation can be repeated after 4 to 6 months, to check the consistency of the findings.

KEY WORDS: brain ageing, dementia, neuropsychological tests.

O envelhecimento normal do cérebro pode se acompanhar de alterações mentais superponíveis às de uma demência incipiente, levando a problemas de diagnóstico diferencial, especialmente com

*Unidade de Neuropsicologia e Neurolinguística, Departamento de Neurologia, Faculdade de Ciências Médicas (FCM) da Universidade Estadual de Campinas (UNICAMP): *Professor Assistente Doutor e Chefe de Departamento. Aceite: 24-outubro-1998.

Dr. Benito P. Damasceno - Departamento de Neurologia, FCM/UNICAMP - Caixa Postal 6111 - 13083-970 Campinas SP - Brasil. FAX 5519788 7990. E-mail: damescen@turing.unicamp.br 
a doença de Alzheimer (DA). Embora se tenham estabelecido, em consenso, critérios neuropsicológicos, patológicos e de neuroimagem para diferenciação entre o normal e o patológico, o diagnóstico dos problemas cognitivos leves de cada cliente idoso continua representando um desafio. Ainda não se descobriu um marcador biológico seguro da DA. A presença do alelo $E-4$ do gene (do cromossoma 19) da apolipoproteína E representa um fator de risco para o desenvolvimento da DA. Entretanto, muitos indivíduos que têm este alelo jamais se tornam dementes, enquanto outros que não o possuem desenvolvem DA. Além disso, qualquer teste laboratorial ou psicológico pode resultar em falso-positivos ou falso-negativos, devido à própria natureza dos fenômenos biológicos e humanos (de gênese ou condicionamento multifatorial). Por isso, na interpretação dos resultados dos testes deve-se levar em conta outras variáveis (que não a lesão) capazes de influenciar no desempenho das tarefas e testes propostos.

O objetivo deste estudo é discutir o problema do diagnóstico diferencial entre envelhecimento cerebral normal e patológico.

\section{RELATO DOS CASOS}

Caso 1. GCAC, 67 anos, casada, normalista, com queixas de insônia, falta de iniciativa e esquecimento, desde há 2 anos. Apática, às vezes nervosa e desesperada, fugindo de casa sem rumo certo, com inquietação noturna. No último ano, idéias de suicídio, desânimo, com crises de choro ou riso imotivados, preocupação exagerada com os filhos e netos, e perguntas perseverativas. Conforme o marido, "muitas vezes ela não sabe se vestir, saindo do banheiro sem roupas". Antecedentes: há 4 anos, sintomas similares, com apatia e episódios de fala desconexa, que duraram 6 meses. No mais, sadia. Exame neurológico normal. A paciente fazia repetidas perguntas e observações inapropriadas, mostrando-se irritadiça, inquieta, com labilidade afetiva (choro fácil) e queixas hipocondríacas. O CAMDEX ${ }^{1}$ e subtestes da bateria de Luria $^{2}$ revelaram déficit de memorização visual e verbal (errando 30\% dos itens) e de resolução de problemas, estando normal a orientação, percepção tátil, auditiva e visuo-espacial, linguagem, fluência verbal (categoria: animais), praxia oral e manual (ideomotora, construcional), interpretação de provérbios e semelhanças. Exames hematológicos, bioquímicos, serológicos e ressonância magnética (RM) cerebral normais. Melhorou com o tratamento antidepressivo, ficando assintomática após um ano. Diagnóstico: provável pseudodemência depressiva.

Caso 2. TMM, 77 anos, viuva, analfabeta, lavradora, com nervosismo, preocupação exagerada com os filhos (adultos), desânimo, pensamentos de morte, inapetência e possíveis alucinações visuais, desde há um ano. Antes sadia. Exame neurológico: leve tremor de ação, presença de reflexo de sucção e discreta redução de reflexos profundos e sensibilidade vibratória distalmente nos membros inferiores. CAMDEX e testes de Luria: total desorientação no tempo, marcante amnésia verbal (errando 50 a 100\% dos itens) e fluência verbal (categoria: animais) prejudicada por abundante perseveração. Investigação laboratorial e tomografia computadorizada cerebral (TC) normais. Tomografia de perfusão cerebral (SPECT): "discreta hipoperfusão dos giros do cíngulo". A paciente melhorou com o tratamento antidepressivo. Diagnóstico: provável pseudodemência depressiva.

Caso 3. MAC, 61 anos, casada, professora de francês, com esquecimento desde há 4 meses. Esquecia facilmente o tópico da conversação. $\mathrm{Na}$ aula, às vezes, tinha "como que um curto-circuito" ou "falha no fio do pensamento", que logo se retomava com certo esforço. Além disso, "perda de vocabulário durante a aula", especialmente na tradução português-francês; problemas motores com as mãos (“... deixo cair as coisas toda hora"), inclusive com a escrita das letras ("mão dura e lenta"). Difícil lembrar-se do local onde punha as coisas. Acordava à noite sem saber sua posição em relação ao quarto ou à porta. E, quando dirigindo o carro na rua, não sabia "para onde exatamente estava indo ou devia ir". Antecedentes: enxaqueca e prolapso de válvula mitral. Negava depressão, mas relatava uma experiência desagradável ocorrida um mês antes do início dos sintomas: quando estava na fila dentro de um banco, este foi invadido por assaltantes armados, os quais obrigaram todos os clientes e funcionários a deitarem no chão. Neste incidente, a paciente teve a coragem de conversar com os assaltantes e pedir calma e obediência aos demais clientes. O CAMDEX e testes de Luria mostraram apenas discreto déficit de memória verbal (aprendizado de lista de 10 palavras). O exame neurológico, a TC e os exames laboratoriais eram normais. SPECT: "discreta hipoperfusão bitemporal e frontal direita, compatível com doença de Alzheimer". No acompanhamento ambulatorial um ano depois, as alterações clínicas e da neuroimagem haviam regredido. As queixas de amnésia, desorientação espacial e as alterações do SPECT são compatíveis com uma DA incipiente, apesar da curta duração da doença, falta de progressão, preservação da consciência dos déficits 
("insight”) e, sobretudo, bom desempenho nos testes neuropsicológicos (muita queixa subjetiva sem alterações objetivas evidentes). Sabemos que algumas formas da DA (por exemplo, sua "variante com corpúsculos de Lewy”) e outras demências podem ter progressão rápida, preservando, em sua fase inicial, o “insight". Entretanto, a paciente melhorou. E a análise retrospectiva do incidente do banco e de suas repercussões psicológicas revelou que ela havia tido, nos meses subsequentes, uma crise reacional leve, com medo infundado, ansiedade e dificuldades para adormecer (os quais ela negligenciava, mostrando-se mais preocupada com os problemas da memória e orientação espacial). O diagnóstico mais provável é o de uma pseudodemência ansiosa e fóbica.

\section{DISCUSSÃO}

\section{Aspectos neuropsicológicos relevantes}

Memória - O padrão de deterioração da memória no velho normal assemelha-se ao encontrado nas fases iniciais da DA: declínio da memória "operacional" e da memória "secundária" ("recente") maior que o da memória "primária" ("imediata") e da memória "terciária" ("remota"). O aprendizado de situações ou informações novas, a evocação retardada e repetição de números em ordem inversa são as funções mnésicas mais alteradas, enquanto o vocabulário, o fundo de informações, a repetição de números em ordem direta e a realização de tarefas rotineiras e automatizadas mantêm-se relativamente intactas. As dificuldades de memória relacionadas à idade são maiores para a memória episódica do que para a memória semântica e pioram em ordem crescente na seguinte sequência: memória de procedimentos, reaprendizado, memória de reconhecimento, evocação baseada em pistas contextuais, evocação livre, memória prospectiva (lembrar de lembrar) ${ }^{3}$. O envelhecimento afeta sobretudo a memória prospectiva e a evocação livre e retardada de material verbal aprendido, preservando sua lembrança baseada em pistas contextuais (p.ex., à base de imagens visuais). Na discriminação entre DA incipiente e envelhecimento normal, os melhores testes têm sido o de evocação retardada (após 15 a 30 minutos) de listas de 10 a 15 palavras aprendidas ${ }^{4}$ e o de memória lógica (recontagem de estórias e recordação de lista de palavras associadas a figuras) $)^{5,6}$.

Função intelectual e visuo-motora-espacial complexa - Pacientes com demência leve apresentam escores inferiores aos de sujeitos normais em testes de formação de conceitos, raciocínio verbal (subteste de semelhanças do WAIS, resolução de problemas), praxias manuais complexas, habilidades visuo-espaciais e práxico-construtivas ${ }^{5,7}$.

Linguagem - De modo geral, a linguagem do idoso saudável ou demente tem sido avaliada com testes metalinguísticos, limitados aos níveis fonológico, sintático e semântico-lexical, dando pouca importância ao nível discursivo-pragmático, que pode mostrar alterações precoces nessas situações. Estes testes não incluem aspectos discursivos e epilinguísticos, deixando assim de detectar perturbações do processo de significação, como as alterações nas relações de sentido, problemas com pressupostos interpretativos, violação de leis conversacionais ou discursivas, dificuldades com operadores argumentativos, alterações de mecanismos de coesão e coerência textual, dificuldades com acesso e manutenção de tópicos.

O envelhecimento normal deixa relativamente intactos o vocabulário e o processamento sintático $^{8}$, enquanto altera a lembrança de palavras (na conversação e em testes de fluência verbal), aparecendo então raras parafasias semânticas. No nível discursivo, podemos ver dificuldades narrativas (especialmente com inferências, sumarização e interpretação moral de estórias) e omissão de informações sobre a "situação" da estória; omissão de passos essenciais durante a descrição de procedimentos; e na conversação, dificuldade de compreensão, falta de clareza do enunciado, "parafasias narrativas" e problemas com inferências e pressuposições ${ }^{8,9}$. Estudos longitudinais utilizando recontagem e interpretação de estórias mostram variação intraindividual da "amnésia" de um dia para o outro, dependendo de variáveis como o tamanho e complexidade da estória, alterações do humor, problemas de família, doenças passageiras, efeito colateral de medicamentos, entusiasmo, familiaridade ou interesse com a estória e efeito da prática e aprendizado?. 
A DA, em sua fase inicial, acompanha-se de (1) problemas semântico-lexicais similares aos encontrados na afasia semântica ou transcortical sensorial, ou seja, o sujeito esquece ou troca palavras, mostrando linguagem elíptica com empobrecimento do vocabulário (especialmente substantivos de baixa frequência e nomes próprios), parafasias semânticas, pleonasmos, excesso de dêiticos e perífrases; e ainda mantém o "insight" (função epilinguística) sobre seus erros ${ }^{10}$. A experiência com o CERAD ${ }^{11}$ aponta o Teste de Nomeação de Boston como o melhor teste não-mnésico para discriminar entre DA e envelhecimento normal. (2) Dificuldades semântico-discursivas na interpretação de metáforas, provérbios, moral de estórias e material humorístico ${ }^{10}$. Nos estágios moderados da DA temos piora destas alterações, aparecendo então violação de leis conversacionais, perda da função epilinguística e início de alterações fonológicas e sintáticas ${ }^{10,12}$.

\section{Neuropatologia}

Com o avançar da idade, particularmente após a sexta década, acelera-se o processo de atrofia cerebral, com dilatação de sulcos e ventrículos, perda de neurônios, presença de placas neuríticas (PN) e emaranhados neurofibrilares (ENF), depósitos de proteína beta-amilóide e degeneração granulovacuolar, os quais aparecem precocemente nas regiões temporais mediais e espalham-se por todo o neocórtex. Na DA, já em suas fases iniciais, estas alterações são mais acentuadas, particularmente a maior densidade de ENF no córtex peri- e entorrinal, subiculum e região CA1 do hipocampo, em correlação com os distúrbios precoces e proeminentes da memória factual ("secundária") observados nesta doença.

$\mathrm{Na}$ histopatologia, os limites entre o normal e o patológico são também imprecisos, daí a necessidade de estabelecer critérios consensuais (tipo de coloração, número de placas neuríticas e emaranhados neurofibrilares por milímetro quadrado de tecido extraído de determinadas regiões cerebrais), levando-se em conta a idade do sujeito. A simples presença dessas alterações nem sempre prediz deterioração neuropsicológica. O diagnóstico neuropatológico deve levar em conta a gravidade dessas alterações, sua localização ou distribuição, a presença de lesões cumulativas prévias (como traumáticas, vasculares, neurotóxicas) e a história prévia de demência. A literatura neuropatológica registra casos diagnosticados clinicamente como "provável” DA, mas sem as alterações patológicas desta doença ${ }^{13}$; e casos de sujeitos idosos cognitivamente intactos que apresentam grande número de placas neuríticas no neocórtex, satisfazendo os critérios diagnósticos de $\mathrm{DA}^{14}$.

\section{Neurofisiologia e neuroimagem}

1. Potenciais evocados relacionados a eventos - O mais usado é a onda $\mathrm{P} 300$, de origem anatômica desconhecida. Na testagem são apresentados dois tons com diferentes probabilidades de ocorrência; e o sujeito tem que contar (ou dar uma resposta motora) ao tom que ocorre de forma infrequente e caótica. A latência do pico da P300 aumenta linearmente com a idade, cerca de 1 a 2 milisegundos/ano. Um retardo na latência desta onda é considerado como medida crucial para o diagnóstico de alterações cognitivas e demência; entretanto, a grande variação interindividual (em torno de 50 milisegundos) tem limitado a confiabilidade clinica da P300 na decisão de cada caso individual; e este aumento de latência não é um índice confiável de disfunção da memória nas fases iniciais da DA, sendo também observado em sujeitos com depressão, esquizofrenia e outros tipos de demência ${ }^{15}$.

2. Eletrencefalograma (EEG) quantitativo e mapeamento cerebral - O EEG de idosos sadios mantém as mesmas caracteristicas gerais do de adultos mais jovens, podendo acompanhar-se de pequenas alterações, como aumento da atividade beta, diminuição da reatividade alfa, além de fragmentação do sono e declínio da porcentagem do sono de ondas lentas ${ }^{16}$. A lentificação generalizada do EEG encontrada em estudos anteriores pode estar associada a variáveis não controladas, como o uso de drogas neurolepticas, antidepressivas e analgésico-sedativas, o nivel de consciência e o estado 
cognitivo-comportamental. Lentificação teta (e delta) na região temporal esquerda é vista em idosos normais (sem correlação com deficits neuropsicológicos) e em pacientes com graus leves a moderados de DA, tornando-se bitemporal ou mais generalizada nos graus severos da demência, em correlação com os sinais de hipometabolismo dessas regiões ${ }^{17}$. Em individuos com "distúrbio da memória associado à idade" ("age-associated memory impairment"), sem outros deficits cognitivos, há uma evidente associação entre o déficit mnésico e a lentificação temporal esquerda. Muitos desses casos podem estar numa fase inicial da DA.

3. Neuroimagem - Em sujeitos idosos sadios, a neuroimagem estrutural com TC e RM mostra redução do volume total do cérebro, com dilatação dos sulcos e sistema ventricular, especialmente dos ventrículos laterais e III ventrículo. Estas alterações são mais acentuadas nos sujeitos dementes quando analisados como grupo, podendo estar ausentes em casos indivíduais com franca demência e presentes em outros cognitivamente intactos. As lesões hiperintensas da substância branca visualizadas nas imagens T2 da RM não têm significado patológico seguro. Mesmo as lesões confluentes e extensas, geralmente associadas a patologia vascular, podem ser vistas em indivíduos idosos sadios (sem sinais de demência vascular) e devem ser interpretadas à luz dos dados da história e do exame clínico-neuropsicológico. Na decisão diagnóstica de casos individuais, o achado de atrofia regional associada a uma síndrome "focal" que corresponda à área afetada pode ser mais relevante que o de uma atrofia cortical generalizada.

Nas fases iniciais da DA (manifestando-se como amnésia), a RM de alta resolução pode mostrar atrofia do hipocampo e do córtex entorrinal (particularmente dos aglomerados celulares da camada II deste cortex); e nos casos negativos, uma RM funcional poderia detectar alterações de fluxo sanguíneo muito antes que tal atrofia se manifeste.

A maioria dos estudos de PET e SPECT com sujeitos idosos sadios mostram redução do metabolismo e fluxo sanguíneo regional cerebral, a qual não se verificou em estudos com sujeitos idosos cuidadosamente selecionados. Na DA, observa-se tipicamente uma redução bilateral e frequentemente assimétrica do fluxo sanguineo e metabolismo em regiões temporais ou têmporoparientais; porém, tais alterações podem estar ausentes nas fases iniciais da doença ou podem ocorrer em outros tipos de demência como a demência vascular e a doença de Parkinson. Por outro lado, a DA e outras demências degenerativas costumam iniciar-se com padrões "focais" (às vezes unilaterais) de hipometabolismo e síndromes cognitivas associadas (a amnésica, a visuo-espacial-apráxica, a afásica e a psíquico-comportamental). Hipometabolismo pré-frontal lateral esquerdo tem sido encontrado na pseudodemência depressiva ${ }^{18}$, às vezes fazendo pensar na doença de Pick. E mesmo estados de ansiedade, estresse ou reduzida acuidade senso-perceptiva durante a realização desses exames podem acompanhar-se de alterações dramáticas do fluxo sanguineo e metabolismo regional do cérebro ${ }^{19}$. Tudo isto deve ser levado em conta no diagnóstico diferencial entre o normal e o patológico, e entre os diversos tipos de demência.

\section{CONCLUSÕES}

No diagnóstico diferencial, os passos mais importantes são os seguintes: (1) anamnese cuidadosa dos problemas atuais e do nível de funcionamento cognitivo e sócio-ocupacional prémórbido, prestando-se bastante atenção ao uso de (poli)fármacos; (2) exame físico e neurológico, buscando sinais sugestivos de doenças sistêmicas associadas (como hipovitaminose B12, hipotiroidismo, etc.) e das doenças neurodegenerativas mais comuns; (3) avaliação neuropsicológica e psíquico-comportamental abrangente (incluindo testes com poder discriminatório, como o de memória retardada), (4) em combinação com RM de alta resolução para a região entorrinal-hipocampal ou outra(s) região(ões) suspeita(s) e técnicas de neuroimagem funcional (em nosso meio, o SPECT); e (5) acompanhamento longitudinal, com retestagem do paciente após 3 a 6 meses, checando-se a consistência dos achados. 


\section{REFERÊNCIAS}

1. Roth M, Tym E, Mountjoy CQ, et al. CAMDEX: a standardised instrument for the diagnosis of mental disorders in the elderly with special reference to the early detection of dementia. Br J Psychiatry 1986;149:698-709.

2. Christensen A-L. Luria's neuropsychological investigation. Copenhagen: Munksgaard, 1979.

3. Craik FIM. Changes in memory with normal aging: a functional view. In Wurtman RJ, Corkin S, Growdon JH, RitterWalker E (eds). Advances in Neurology Vol. 51, Alzheimer's Disease. New York: Raven Press, 1990:201-205.

4. Welsh KA, Butters N, Hughes JP, Mohs RC, Heyman A. Detection and staging of dementia in Alzheimer's disease. Arch Neurol 1992;49:448-452.

5. Erkinjuntti T, Laaksonen R, Sulkava R, Syrjalainen R, Palo J. Neuropsychological differentiation between normal aging, Alzheimer's disease and vascular dementia. Acta Neurol Scand 1986;74:393-403.

6. Howieson DB, Dame A, Camicioli R, Sexton G, Payami H, Kaye JA. Cognitive markers preceding Alzheimer's dementia in the healthy oldest old. J Am Geriatr Soc 1997;45:584-589.

7. Jacobs DM, Sano M, Dooneief G, Marder K, Bell KL, Stern Y. Neuropsychological detection and characterization of preclinical Alzheimer's disease. Neurology 1995;45:957-962.

8. Ulatowska HK, Cannito MP, Hayashi MM, Fleming SG. Language abilities in the elderly. In Ulatowska HK (ed.). The aging brain: communication in the elderly. London: Taylor \& Francis, 1985:125-139.

9. Dixon RA, Hertzog C, Friesen IC, Hultsch DE. Assessment of intraindividual change in text recall of elderly adults. In Brownell HH, Joanette Y (eds.), Narrative discourse in neurologically impaired and normal aging adults. San Diego: Singular Publ Group 1993:77-101.

10. Bayles KA, Kaszniak AW. Communication and cognition in aormal aging and dementia. Boston: Little Brown, 1987.

11. Morris JC, Heyman A, Mohs RC, et al., and the CERAD investigators. The Consortium do Establish a Registry for Alzheimer's Disease (CERAD): Part I. Clinical and neuropsychological assessment of Alzheimer's disease. Neurology 1989;39:1159-1165.

12. Coudry MIH, Morato E. Alterações neurolinguísticas na demência do tipo Alzheimer. II Congresso da Associação Brasileira de Psiquiatria Biológica, 1990:1-6.

13. Heilig CW, Knopman DS, Mastri AR, Frei II W. Dementia without Alzheimer pathology. Neurology 1985;35:762-765.

14. Katzman R, Terry R, DeTeresa R, et al. Clinical, pathological, and neurochemical changes in dementia: a subgroup with preserved mental status and numerous neocortical plaques. Ann Neurol 1988;23:138-144.

15. DeToledo-Morrell L, Morrel F. Alzheimer's disease: new developments for noninvasive detection of early cases. Curr Opin Neurol Neurosurg 1993;6:113-118.

16. Ehlers CL, Kupfer DJ. Effects of age on delta and REM sleep parameters. Electroencephalogr Clin Neurophysiol 1989;72:118-125.

17. Evans WJ, Starr A. Electroencephalography and evoked potentials in the elderly. In Albert ML, Knoefel JE (eds.). Clinical neurology of aging. New York: Oxford Univ Press, 1994:235-265.

18. Baxter LR, Schwartz JC, Phelps ME, et al. Reduction of prefrontal cortex glucose metabolism common to three types of depression. Arch Gen Psychiatry 1989;46:243-250.

19. Gur RC, Gur RE, Resnick SM, Skolnick BE, Alavi A, Reivich M. The effect of anxiety on cortical cerebral blood flow and metabolism. J Cer Blood Flow Metab 1987;7:173-177. 